\title{
Análise das metas de controle da tuberculose no Brasil: uma revisão narrativa
}

\author{
Analysis of tuberculosis control goals in Brazil: a narrative review
}

Análisis de los objetivos de control de latuberculosisen Brasil: una revisión narrativa

Kelly de Oliveira Galvão da Silva ${ }^{1 *}$, Maikiane Aparecida Nascimento ${ }^{1}, V^{2}$ léria Moura de Carvalho', Gabriela Cavalcante Oliveira ${ }^{1}$, Ailton José Soares ${ }^{1}$, Andrea Cândida dos Santos Furtado', Marielly Sousa Borges ${ }^{1}$, Jefferson do Carmo Dietz ${ }^{1}$, Nayara Messias da Silva ${ }^{1}$.

\section{RESUMO}

Objetivo: Destacar as metas de controle da tuberculose e os indicadores epidemiológicos, que serão monitorados a cada triênio a partir de 2017, e traçar um paralelo entre as metas que já foram e as que devem ser alcançadas. Revisão Bibliográfica: O país, apesar de ser referência mundial no controle da tuberculose e ter alcançado antecipadamente as metas dos Objetivos de Desenvolvimento do Milênio antes de 2015, ainda não alcançou as metas do End TB, contudo vem demostrando redução nos indicadores. Assim, a meta da nova estratégia de enfrentamento da tuberculose é que, até 2035, o mundo esteja livre da doença. Para isso, medidas que versam sobre atenção ao paciente, componente social e pesquisa e inovação devem ser adotadas, sendo este tema prioritário, de acordo com o Plano Nacional de Promoção de Saúde na busca da erradicação do agravo. Considerações finais: Diante disso, é fundamental que haja o aprimoramento na educação permanente dos profissionais da área da saúde a fim de alcançar as metas propostas.

Palavras-chave: Tuberculose, Saúde pública, Metas.

\begin{abstract}
Objective: To highlight the tuberculosis control goals and the epidemiological indicators that will be monitored every three years starting in 2017 and to draw a parallel between the goals that have already been and should be achieved. Bibliographic Review: The country, despite being a world reference in the control of tuberculosis and having previously achieved the goals of the Millennium Development Goals before 2015, has not yet reached the goals of End TB, however it has been showing a reduction in indicators. Thus, the goal of the new strategy to fight tuberculosis is that by 2035 , the world will be free of the disease. For that, measures that deal with patient care, social component and research and innovation must be adopted. This topic being a priority, according to the National Health Promotion Plan in the quest to eradicate the disease. Final considerations: Thereby, it is essential to improve the continuing education of health professionals in order to achieve the proposed goals.
\end{abstract}

Keywords: Tuberculosis, Public health, Goals.

\section{RESUMEN}

Objetivo: Destacar los objetivos de control de la tuberculosis y los indicadores epidemiológicos, que se supervisarán cada tres años a partir de 2017, y establecer un paralelismo entre los objetivos que ya se han alcanzado y los que se deben alcanzar. Revisión bibliográfica: El país, a pesar de ser una referencia mundial en el control de la tuberculosis y haber alcanzado previamente los objetivos de los Objetivos de Desarrollo del

${ }^{1}$ Laboratório Estadual de Saúde Pública Dr. Giovanni Cysneiros - LACEN/GO, Goiânia- GO.

*E-mail:k.galvao@yahoo.com.br. 
Milenio antes de 2015, aún no ha alcanzado los objetivos del End TB para poner fin a la tuberculosis, sin embargo, ha mostrado una reducción en los indicadores. Por lo tanto, el objetivo de la nueva estrategia para combatir la tuberculosis es que, para 2035, el mundo estará libre de la enfermedad. Para eso, se deben adoptar medidas que aborden la atención al paciente, el componente social y la investigación e la innovación. Este tema es una prioridad, de acuerdo con el Plan Nacional de Promoción de la Salud, en la búsqueda de erradicar la enfermedad. Consideraciones finales: En vista de esto, es esencial mejorar la educación continua de los profesionales de la salud para lograr los objetivos propuestos.

Palabras clave: Tuberculosis, Salud pública, Objetivos.

\section{INTRODUÇÃO}

Vários vestígios fósseis demonstraram que, há milhares de anos, a tuberculose (TB), uma doença infectocontagiosa, vem afetando humanos e animais. O agente etiológico foi caracterizado por Koch, em 1882, e sua identificação possibilitou melhor compreensão da doença, abrindo caminhos para a implementação de novos tratamentos para a doença (MARQUES AMC e CUNHA RV, 2003).

$\mathrm{Na}$ tríade epidemiológica da doença, sabe-se que elementos não biológicos também são determinantes da morbimortalidade desse agravo. Fatores de cunho social e econômico, tais como, pobreza extrema, baixa escolaridade, saneamento básico precário, são algumas das condições que favorecem o desenvolvimento e o agravamento dos quadros da doença (OLIVEIRA NF e GONÇALVES MJF, 2013). Nesse cenário, intervenções nas condições de vida para modificar os alarmantes indicadores da tuberculose são fundamentais (RUFFINO-NETTO A, 2002).

As estratégias brasileiras tomadas para o controle da patologia de 1990 a 2015 obtiveram sucesso. Notase, em determinado momento, a redução em $42 \%$ e em $47 \%$, respectivamente, na prevalência da doença e na mortalidade (WORLD HEALTH ORGANIZATION, 2016).

As melhorias na infraestrutura dos países, os avanços no tratamento com a descoberta dos primeiros antibióticos e o aprimoramento das técnicas de diagnóstico, associados a maciços investimentos e doações internacionais, contribuíram significativamente na redução dos indicadores (ANTUNES JLF, et al., 2000).

Vários fatores geram preocupações, além da gravidade da doença, como a taxa de abandono do tratamento, que é em torno de $12 \%$, o que é preocupante devido às implicações negativas a que leva esta prática (SOUZA KMJ, et al., 2010).

Outro obstáculo são as resistências, que na maioria das vezes são geradas devido à utilização de medicamentos de forma inadequada, assim como nos casos de abandono, que pode ocorrer o aparecimento de resistência às drogas, pois a cada reinício de uso poderá haver a seleção dos mutantes resistentes devido às diferenças nos tempos de ação de cada droga (SEVERO NPF, et al., 2007).

Diante disso, medidas de controle da tuberculose são extremamente necessárias. Para isso, a articulação de estratégias que vão além da vigilância epidemiológica, que abarquem diagnósticos precoces e precisos, identificação correta do desenvolvimento de resistência bacteriana, tratamentos rápidos e efetivos para a TB sensível, como também para a resistente, medidas profiláticas eficientes como a imunização e o tratamento de bacilíferos ativos, políticas de proteção social e suporte aos doentes são imprescindíveis (BARREIRA D, 2018).

Em nível mundial, a estratégia End TB pelo Fim da Tuberculose concebida pela Organização Mundial da Saúde (OMS) em 2014, propõe uma mudança radical de paradigma na luta contra a TB, com o objetivo de eliminar a doença como problema de saúde pública: reduzir em $90 \%$ os casos de TB, e reduzir em $95 \%$ as mortes por TB, e coeficiente de incidência menor que 10 casos/100 mil habitantes até 2035 (WHO, 2014).

Dessa forma, o objetivo desse estudo é realizar uma breve descrição histórica da tuberculose e destacar as medidas e metas de controle propostas na estratégia End TB, traçando um paralelo entre as que já foram e as devem ser alcançadas. 


\section{REVISÃO BIBLIOGRÁFICA}

\section{Breve histórico da tuberculose}

Os primeiros casos de TB em humanos fazem menção a dados históricos há 5.000 anos a.C. No Brasil, a chegada desse agravo reporta-se ao aparecimento de casos após a colonização portuguesa. Estudiosos teorizam que jesuítas e colonos já infectados transmitiam a infecção para aqueles que tinham contatos com eles. Vários estudos citam que o registro do primeiro caso de portador da doença no Brasil ocorreu com o Padre Manuel da Nóbrega, em 1549, que foi a óbito pela doença (CAMPOS R e PIANTA C, et al., 2001).

Há inclusive relatos de que indígenas catequisados adoeciam apresentando sintomas sugestivos de tuberculose nos escritos de José de Anchieta (1583) e Inácio Loyola (1555). São relatados achados como tosse, escarro sanguinolento e óbito na maioria das vezes (RUFFINO-NETTO A e PEREIRA JC, 1981; ROSEMBERG J, 1999; MACIEL ELN, 2016).

Semelhante a países da Europa durante a Revolução Industrial, a epidemia da TB se alastrava no Brasil e denominava-se a patologia como a "praga dos pobres", associando-a às pessoas acometidas, que proviam, em sua maioria, de habitações em locais impróprios, com condições insalubres, vivendo em aglomerados de pessoas em pequenos espaços, com falta de higiene e alimentação precária (SHEPPARD DS, 2001).

A situação incomodava os governantes e a população, gerando inquietude e estimulando a busca de uma resolução do problema. Vários decretos e avisos ministeriais estabeleciam novas regras e instituíam questões sanitárias com o objetivo de conter o agravo, porém a saúde pública era norteada pelo interesse econômico e político da elite (ANTUNES JLF, et al., 2000).

Atualmente esse agravo ainda é endêmico no país, sendo encontrado em grandes centros urbanos, em aglomerados de pessoas e principalmente em populações mais vulneráveis, como os privados de liberdade e os portadores de alguma imunossupressão. Desse modo, o Brasil faz parte do grupo dos 30 países priorizados pela OMS e ocupa a $20^{\underline{a}}$ posição em número absoluto de casos de TB e a $19^{\mathrm{a}}$ em casos de coinfecção de Tuberculose e Vírus da Imunodeficiência Humana (HIV) (BRASIL, 2017a).

A TB é um agravo de notificação compulsória, o qual configura um grave problema de saúde pública, responsável por vários casos de morbimortalidade da população até nos dias atuais, e, mesmo fazendo parte das prioridades na agenda do Ministério da Saúde (MS), observa-se que há muitas iniquidades no acesso ao diagnóstico e ao tratamento, fato evidenciado pelos números de notificações de casos novos e óbitos a cada ano (WHO, 2017).

Segundo a OMS, em seu último relatório (2019), estima-se que aproximadamente 10,0 milhões de pessoas adoeceram de TB em todo o mundo, em algumas regiões, o coeficiente de incidência supera 500 casos/100 mil habitantes/ano, e o coeficiente global ainda é de 130 casos/100 mil habitantes/ano. Cerca de 1,2 milhões de pessoas que não possuíam HIV morreram da doença, uma redução de $27 \%$ comparada a 2017 . Houve um acréscimo de 251.000 mortes entre as pessoas vivendo com HIV (WHO, 2019).

Em 2018, o Brasil ocupava o 14\% lugar entre os 22 países responsáveis por $80 \%$ do total de casos de tuberculose no mundo. A prevalência estimada no país era de 5 milhões de infectados, aproximadamente 109,672 casos novos e cerca de 6.000 óbitos por ano.

Segundo o MS, no ano de 2018 foram diagnosticados 72.788 casos novos de TB, cuja taxa de incidência foi de 34,8 casos $/ 100$ mil habitantes e o coeficiente de mortalidade 2,2 /100 mil habitantes. Houve maior predomínio nos estados do Amazonas, com 72,9 casos/100 mil habitantes e Rio de Janeiro com 66,3 casos/100 mil habitantes (BRASIL, 2019a).

\section{Tuberculose rumo ao controle}

A alta incidência e a crescente taxa de mortalidade estimularam ainda mais a busca de um plano de ação, junto ao setor público, que pudesse controlar essa patologia. Dessa forma, no final da década de 1940, houve redução dos indicadores de TB, tendo em vista a implementação do tratamento quimioterápico e das ações de combate ao agravo (MISHIMA EO e NOGUEIRA PA, 2001). Desde então, sucessos e fracassos marcaram a história da TB no Brasil. 
Na década de 1970, no Brasil, buscava-se programar ações a fim de um controle efetivo da TB. Com o surgimento do Sistema Único de Saúde (SUS), lentamente começou-se a organizar o Programa Nacional de Controle da Tuberculose (PNCT), cujos princípios seriam voltados para o diagnóstico e o tratamento precoce dos indivíduos com TB; a prevenção da doença por meio da vacinação com a vacina Bacillus Calmette-Guérin (BCG); além de priorizar as populações vulneráveis (CYRIACO C, et al., 2014).

Com este mesmo intuito, foi também formada a Rede Brasileira de Pesquisas em Tuberculose (REDETB), com a finalidade de promover a interação entre as esferas política, científica e industrial para a cooperação no desenvolvimento de políticas capazes de controlar a tuberculose no país.

Desde então, a rede vem se fortalecendo na luta contra a TB e novos projetos foram incorporados, dentre eles destacam-se os testes moleculares nas abordagens diagnóstica, terapêutica e suas resistências. Logo, com o monitoramento dos casos novos, retratamentos e das tuberculoses extrapulmonares, espera-se alcançar metas de sucesso em curto e médio prazo. Nessa proposta, incluiu-se a interação da promoção de saúde e a pesquisa operacional, nas quais se tornou crucial a mediação do SUS (KRITSKI A, et al., 2016).

Somando a isso, a descentralização do programa de controle atribuiu responsabilidade específica para cada esfera de gestão, a autonomia de cada uma delas, e iniciativas políticas, tornando-as altamente independentes, porém interligadas pelos princípios e diretrizes do SUS. O PNCT norteia as ações nos municípios brasileiros, e, para isso, é necessária a participação e a atuação das esferas federal, estadual e municipal (PEREIRA AA, et al., 2016).

A gravidade do problema é reconhecida pelas autoridades competentes do Brasil. Em razão disso, os propósitos de suas políticas públicas assumiram o compromisso com seus cidadãos e com a comunidade internacional de controlar a evolução da doença, procurando reduzir sua prevalência na população, sendo assim notados grandes avanços com o PNCT, mas ainda existem vários desafios a seguir (PINTO ESG, et al., 2018).

O progresso alcançado é fruto do conjunto de atividades integradas entre pesquisadores, governo e sociedade civil. Haja vista que algumas áreas se encontram mais evoluídas que outras, porém a missão da rede é interligar governos, academias da sociedade civil organizada e a indústria em prol do controle da TB (MACIEL ELN, 2016).

No Brasil, há alguns anos, foi lançado pelo Ministério da Saúde o PNCT, que tinha como metas prioritárias para o controle da TB até 2007: média anual de detecção de pelo menos $70 \%$ dos casos, curar pelo menos $85 \%$, taxa de abandono menor que $5 \%$, supervisionar o tratamento em $100 \%$ dos casos nos municípios prioritários e ampliar para 100\% o número de bacilíferos examinados (BRASIL, 2004). Posteriormente, foi lançada pela OMS uma ousada meta de eliminação da tuberculose no mundo até 2035.

Como resultado deste programa, observou-se uma redução da mortalidade no país de aproximadamente 42\% no período de 1982 a 2002. Tal declínio pode ser explicado pela melhor atenção ao doente, pelo desenvolvimento de medicamentos mais eficientes, pela criação do SUS e, é evidente, por uma melhora nas condições de vida da população brasileira (BARREIRA D, 2018).

\section{Metas e estratégias para o enfrentamento da Tuberculose}

No Quadro 1 é apresentado o Panorama das estratégias acordadas mundialmente para alcançar as metas para eliminação da tuberculose até 2035. Cabe ressaltar, que o Brasil tem se destacado como refencia global na articulação de politicas para o alcance das metas estipuladas. 
Quadro 1 - Panorama das estratégias para alcançar as metas para eliminação da tuberculose até 2035.

\begin{tabular}{|c|c|}
\hline Autor, ano & Panorama das estratégias para alcançar as metas para eliminação da tuberculose até 2035 \\
\hline Barreira D, 2018 & $\begin{array}{l}\text { Estabelecida a meta de detectar os } 4,1 \text { milhões de casos não diagnosticados globalmente. } \\
\text { Brasil foi citado como referência global do controle da TB com políticas públicas, como SUS e Bolsa-família. } \\
\text { Em } 2011 \text {, o Brasil alcançou as metas dos Objetivos de Desenvolvimento do Milênio antes do prazo estabelecido } \\
\text { pela Organização das Nações Unidas (ONU). }\end{array}$ \\
\hline Maciel ELN, 2016 & $\begin{array}{l}\text { Foi pactuada a criação da REDE-TB para cooperação científica entre pesquisadores da tuberculose. } \\
\text { Estipularam-se metas mundiais de redução da incidência para menos de } 10 \text { casos por } 100 \text { mil habitantes e redução } \\
\text { da mortalidade pela doença em } 95 \% \text { até } 2035 \text {. } \\
\text { Foram destacados os avanços no diagnóstico, como a implantação do teste rápido molecular para TB (TRM-TB), } \\
\text { bem como detecção de resistência à rifampicina. }\end{array}$ \\
\hline Maciel ELN, 2018 & $\begin{array}{l}\text { Intensificação da estratégia Tratamento Diretamente Observado (TDO), que propicia redução das taxas de } \\
\text { abandono, morte e desenvolvimento de TB multidrogarresistente (TB-MDR). } \\
2009 \text { - Implantação de novo protocolo de tratamento com rifampicina, isoniazida, pirazinamida e etambutol (RHZE) } \\
\text { em uma formulação de combinação de dose fixa (FDC): RHZE-FDC como importante fator para a melhora no } \\
\text { controle da doença. }\end{array}$ \\
\hline Kritski A, 2016 & $\begin{array}{l}\text { Na Conferência Ministerial Global da OMS pelo Fim da TB, em novembro de } 2017 \text {, foi aprovada a Declaração de } \\
\text { Moscou para Erradicar a TB. } \\
\text { Proposta de estratégias integradas para o estudo e controle da tuberculose no Brasil: novas drogas, novas vacinas, } \\
\text { testes diagnósticos e avaliação clínico-operacional. }\end{array}$ \\
\hline Silva DR, 2019 & $\begin{array}{l}\text { Na América Latina, a incidência diminui } 1,7 \% \text { ao ano desde } 2000 \text {. } \\
\text { A produção científica se tornou maior no Brasil, México e Peru. }\end{array}$ \\
\hline
\end{tabular}

Fonte: SILVA KOG, et al., 2020.

REAS/EJCH | Vol.12(5) | e3063 | DOI: https://doi.org/10.25248/reas.e3063.2020 Página $\mathbf{5}$ de $\mathbf{1 0}$ 
Atualmente, a meta proposta como nova estratégia de enfrentamento preconiza que, até 2035, o mundo esteja livre da TB, com a incidência atingindo menos de 10 casos por 100 mil habitantes e com o número de óbitos reduzido em 95\% (BRASIL, 2019a).

O Brasil alcançou as metas dos Objetivos de Desenvolvimento do Milênio (ODM) para o controle da TB antes de 2015. A meta de reduzir pela metade a taxa de mortalidade por TB quando comparada a 1990 foi alcançada em 2011, com três anos de antecedência, e o número de óbitos notificados, próximo de 4.600, correspondendo a 2,4 óbitos por 100 mil habitantes também esteve dentro da meta proposta (MACIEL ELN, et al., 2018). Contudo, o país ainda não conseguiu atingir as principais metas estabelecidas na estratégia nos últimos 20 anos, conforme demonstrado (Tabela 1), algumas metas, como redução do coeficiente de incidência e o aumento da taxa de tratamento diretamente observado (TDO), estão longe de serem atingidas.

Tabela 1 - Comparação da meta 2035 aos indicadores alcançados pelo Brasil, no ano 2001, no intervalo de 2008 a 2015 , e nos anos 2016 a 2018

\begin{tabular}{ccccccccc}
\hline Indicadores & Meta 2035 & \multicolumn{4}{c}{ Indicadores alcançados pelo Brasil } & Status da meta \\
& 2001 & $\begin{array}{c}2008- \\
2015\end{array}$ & 2016 & 2017 & 2018 & \\
\hline $\begin{array}{c}\text { Coeficiente de } \\
\text { incidência } \\
\begin{array}{c}\text { Coeficiente de } \\
\text { mortalidade }\end{array}\end{array}$ & 10,0 & 47,2 & 36,7 & 34,0 & 33,5 & 34,8 & Necessita redução de $2 / 3$ \\
$\begin{array}{c}\text { Taxa cura } \\
\text { Taxa de }\end{array}$ & 0,0 & 3,1 & 2,7 & 2,0 & 2,1 & 2,2 & Muito próximo da meta \\
$\begin{array}{c}\text { abandono } \\
\text { Taxa de TDO }\end{array}$ & 100 & 72,2 & 70,0 & 61,4 & 73,0 & 71,4 & Próximo da meta \\
\hline
\end{tabular}

Legenda: SI: Sem informação. Valores de coeficiente de incidência e mortalidade expressos em $n^{\circ}$ de casos/100 mil habitantes; valores de taxa de cura, abandono e TDO expressos em percentuais (\%).

Fonte: Silva KOG, et al., 2020; dados extraídos do relatório do Programa Nacional de Controle da Tuberculose (PNCT) e do Boletim Epidemiológico da tuberculose de 2017 e 2018.

Em relação às metas, embora não atingidas em sua totalidade, melhoraram significativamente alguns parâmetros, por exemplo, o tratamento supervisionado ampliou-se de $3 \%$, em 1998, para até $68 \%$, em 2005, em algumas localidades; os índices de cura atingiram 79\% e a taxa de abandono manteve-se em até 2,2\% em algumas regiões para pacientes em Tratamento Diretamente Observado (TDO) (BRASIL, 2011).

Já no final de 2006, foi lançado um novo programa pelo MS, denominado Plano Estratégico para o Controle da Tuberculose, Brasil 2007-2015. Como principais metas, o plano tinha por objetivo: diminuir a incidência de TB, detectar $70 \%$ dos casos infectantes e curar $85 \%$ desses casos, utilizando estratégia TDO, e conseguir a redução à metade da prevalência e mortalidade pela tuberculose em 2015 (WHO, 2017).

Como resultado desse novo programa, observou-se redução de $11,5 \%$ no coeficiente da mortalidade, comparando-se resultados de 2015, com 2,3/100 mil habitantes, e 2006, que apresentou coeficiente de mortalidade de 2,6/100 mil habitantes. Em 2015, também se verificou um aumento no percentual de cura de casos novos pulmonares confirmados por critério laboratorial, atingindo $73,0 \%$, maior valor encontrado desde a implantação do PNCT (BRASIL, 2017b).

Desde 2007, o coeficiente de incidência vem apresentando variação anual média de aproximadamente 1,2\% ao ano. Em 2007, a incidência atingiu a marca de 37,9/100 mil habitantes, já em 2016, esse valor reduziu para 33,7/100 mil habitantes (WHO, 2017). Em relação ao abandono, em 2016, no país, o percentual foi $10,3 \%$, duas vezes acima da meta preconizada pela OMS $(<5,0 \%)$. Porém, três estados alcançaram a meta: Acre $(4,1 \%)$, Tocantins $(4,3 \%)$ e Piauí $(4,6 \%)$. No que se refere às capitais, Porto Velho, Porto Alegre e 
Florianópolis obtiveram percentuais superiores a $20 \%$ de abandono de tratamento. Em 2017, 69.569 casos novos e 4.426 óbitos por tuberculose foram registrados. Registrou-se uma redução média anual de 1,6\%, comparando-se 2016 com 2017 (BRASIL, 2017a; BRASIL, 2019a).

Segundo o boletim epidemiológico do Ministério da Saúde (MS) (2018), em 2017, no Brasil, 71,4\% dos casos novos tiveram diagnóstico por confirmação laboratorial e, nesse cenário, destacam os estados de Roraima (91,5\%), Acre (88,7\%), Espírito Santo (87,5\%) e Amapá (88,5\%). Entretanto, outros estados e capitais tiveram um percentual menor que $60 \%$ de confirmação diagnóstica (BRASIL, 2018).

Em 2017, 71,4\% dos casos novos foram curados, mas apenas $36,9 \%$ realizaram o tratamento diretamente observado. Embora longe da meta, o TDO ampliou-se significativamente nos últimos anos, saindo de um patamar de 3\%, em 1991, e 11,5\%, em 2011, para 36\% em 2018. Tal ampliação é resultado de maciços investimentos no campo da capacitação de profissionais de saúde, em especial os agentes comunitários de saúde, e o fortalecimento da atenção básica (JUNGES JR, et al., 2019; BRASIL, 2011).

Foram notificados 72.788 casos novos de Tuberculose no Brasil, isso representa um aumento discreto, embora a incidência tenha diminuído em torno de 1\% ao ano, esse fenômeno não foi observado em 2018. Esse aumento foi atribuído pela incorporação na rede pública do Teste Rápido Molecular (TRM-TB), o Teste Xpert MTB/RIF, para o diagnóstico de casos novos de TB (BRASIL, 2015).

Esse ensaio clínico trouxe maior eficiência, por ser simples, rápido e de fácil execução, capaz de detectar o Micobacteriumtuberculosis. Além disso, a técnica permite identificar a possível resistência à rifampicina em aproximadamente duas horas. Segundo os documentos da rede, no país já foram realizados 146.939 testes rápidos moleculares, compondo uma média de 12.245 testes por mês (BRASIL, 2011).

O diagnóstico precoce da tuberculose é o primeiro componente do pilar da End TB Strategy da OMS, sendo destacados os avanços no diagnóstico como a implantação do TRM-TB, bem como a detecção de resistência à rifampicina (MACIEL ELN, 2016). Dessa forma, o investimento em pesquisa e capacitação desses profissionais melhorou o processo de trabalho da equipe do laboratório, das vigilâncias estaduais e municipais e acelerou o início do tratamento dos pacientes (MACIEL ELN, et al., 2018).

Desde 2009, o coeficiente de incidência vem apresentando queda anual de aproximadamente $1,0 \%$, entretanto, nos anos de 2017 e 2018, observou-se um pequeno aumento na incidência nacional da tuberculose (BRASIL, 2019a). Nessa projeção, no ano de 2035, o coeficiente de incidência de casos novos de tuberculose seria de 20,7/100 mil habitantes. No entanto, para o alcance dos primeiros objetivos da estratégia End TB, faz-se necessário uma redução média anual de $4 \%$ a $5 \%$ da incidência da TB até 2020 (BRASIL, 2017b). Para tanto, a OMS (2016) prevê uma estratégia tripé para o alcance das metas, com a atuação em três pontos cruciais: atenção ao paciente, componente social, pesquisa e inovações (BRASIL, 2004).

Desse modo, um componente desse tripé, como a atenção ao paciente, situa-se no campo da prevenção e cuidado integrados, sendo proporcionado pelo diagnóstico precoce da doença, com oferta universal de cultura e teste de sensibilidade, incluindo o uso de testes rápidos. Também visa ao tratamento agilizado no contexto da integralidade do cuidado, o que inclui intensificar as atividades colaborativas TB-HIV e as ações de prevenção. (BARREIRA D, 2018; MACIEL ELN, 2016; SOUZA EP, et al., 2015). O componente social fomentará ações para garantir recursos adequados, fortalecendo as articulações intra e intersetorial, e a participação da sociedade civil nas estratégias de enfrentamento da doença, além de melhorar os sistemas informatizados de registros de casos para tomada de decisão oportuna (SANTOS FILHO ET e GOMES ZMS, 2007).

Com relação à pesquisa, o intuito é de estabelecer parcerias para fomentar a realização de pesquisas no país em temas de interesse para saúde pública e promover a incorporação de iniciativas inovadoras para aprimorar o controle da TB (KRITSKI A, et al., 2016). Assim, a cada triênio de 2017 a 2035 serão avaliados os indicadores do programa e seus resultados em cada esfera de gestão, que servirão de subsídio para o desenvolvimento das fases subsequentes. Portanto, espera-se, por meio dessa prática, estimular o profissional ao aperfeiçoamento de habilidades e de aprendizagem a fim de somar ao processo. 


\section{Indicadores de impacto, resultado e processo pelos programas de controle da tuberculose}

No manual "Brasil Livre da Tuberculose" (BRASIL, 2017a), encontra-se a sugestão da tabela de indicadores de impacto, resultado e processo, a serem monitorados pelos programas de controle da TB (Quadro 2).

Quadro 2 - Brasil livre da Tuberculose: indicadores de impacto, resultado e processo a serem monitorados pelos programas de controle da tuberculose no Brasil a partir de 2017.

\begin{tabular}{|c|c|c|}
\hline Tipo & Indicador & $\begin{array}{c}\text { Período de } \\
\text { monitoramento } \\
\text { trienal a partir de } 2017\end{array}$ \\
\hline \multirow{2}{*}{ Impacto } & Coeficiente de incidência de tuberculose todas as formas & Anual \\
\hline & Coeficiente de mortalidade por tuberculose & Anual \\
\hline \multirow{10}{*}{ Resultados } & $\begin{array}{l}\text { Percentual de casos novos pulmonares confirmados por } \\
\text { critério laboratorial }\end{array}$ & Semestral \\
\hline & $\begin{array}{l}\text { Percentual de casos de tuberculose notificados no Sinan } \\
\text { com pós-óbito. }\end{array}$ & Semestral \\
\hline & $\begin{array}{l}\text { Proporção de realização de cultura de escarro entre os } \\
\text { casos de retratamento de tuberculose }\end{array}$ & Semestral \\
\hline & $\begin{array}{l}\text { Proporção de realização de teste de sensibilidade entre os } \\
\text { casos de retratamento de tuberculose com cultura de } \\
\text { escarro positiva }\end{array}$ & Semestral \\
\hline & $\begin{array}{l}\text { Proporção de contatos examinados dos casos novos de } \\
\text { tuberculose pulmonar com confirmação laboratorial }\end{array}$ & Semestral \\
\hline & $\begin{array}{l}\text { Proporção de testagem para HIV entre os casos novos de } \\
\text { tuberculose }\end{array}$ & Trimestral \\
\hline & $\begin{array}{l}\text { Proporção de realização de terapia antirretroviral (TARV) } \\
\text { entre os casos novos de coinfecção TB-HIV }\end{array}$ & Trimestral \\
\hline & $\begin{array}{l}\text { Proporção de casos novos de tuberculose pulmonar que } \\
\text { realizaram o tratamento diretamente observado }\end{array}$ & Trimestral \\
\hline & $\begin{array}{l}\text { Proporção de cura de casos novos de tuberculose } \\
\text { pulmonar com confirmação laboratorial }\end{array}$ & Trimestral \\
\hline & $\begin{array}{l}\text { Proporção de cura de casos novos de tuberculose } \\
\text { multidrogarresistente }\end{array}$ & Anual \\
\hline \multirow{2}{*}{ Processo } & $\begin{array}{l}\text { Indicadores relacionados à tuberculose presentes no plano } \\
\text { de saúde }\end{array}$ & Anual \\
\hline & $\begin{array}{l}\text { Número de estados com comitê de controle da tuberculose } \\
\text { em funcionamento }\end{array}$ & Anual \\
\hline
\end{tabular}

Fonte: Silva KOG, et al., 2020; dados extraídos da Coordenação geral do Programa Nacional de Controle da Tuberculose.

Vale ressaltar que cada indicador possui um período estipulado para ser analisado (trimestral, semestral e/ou anual). Logo, a cada triênio, a tabela será alimentada, a fim de monitorar o agravo e melhorar as tomadas de decisões em cima de cada indicador, para posteriormente alcançar a erradicação da TB no Brasil. Assim, graças ao SUS e sua rede de pesquisas em TB, os dados são evidenciados e são dadas visibilidades às necessidades que ainda existem no sistema. Para isso, faz-se necessária qualificação periódica, monitoramento e avaliação por meios das três esferas do SUS, a fim de ampliar o acesso dessa ferramenta à população (BARREIRA D, 2018). 
Diante dessas metas cabe uma boa gestão, a fim de observar os desafios e diagnosticar os impactos, priorizando o que fazer e o que colocar em prática, pois vários estudiosos afirmam que apenas $10 \%$ das estratégias formuladas chegam ao êxito, pois $90 \%$ se perdem na má execução (KAPLAN RS e NORTON DP, 2000).

\section{CONSIDERAÇÕES FINAIS}

Dessa forma, para atingir as metas estabelecidas pela OMS, torna-se necessário o apoio da promoção da saúde, que respalda a educação permanente dos profissionais de saúde, em benefício da população como primeiro tema prioritário, que, segundo o Plano Nacional de Promoção de Saúde, é de extrema importância na conquista da erradicação desse agravo. Com isso, posteriores estudos poderão comprovar o alcance das metas.

\section{REFERÊNCIAS}

1. ANTUNES JLF, et al. A tuberculose através do século: ícones canônicos e signos do combate à enfermidade. Ciência \& Saúde Coletiva, 2000; 5:367-379.

2. BARREIRA D. Os desafios para a eliminação da tuberculose no Brasil. Epidemiologia e Serviços de Saúde, 2018; $27: \mathrm{e} 00100009: 1-4$.

3. BRASIL. MINISTÉRIO DA SAÚDE. Programa Nacional de Controle da Tuberculose. Brasília: MS, 2004. 28 p.

4. BRASIL. MINISTÉRIO DA SAÚDE. Agenda Estratégica: 2011-2015. Brasília: MS, 2011.

5. BRASIL. MINISTÉRIO DA SAÚDE. Boletim Epidemiológico- Implantação do Plano Nacional pelo Fim da Tuberculose como problema de saúde pública no Brasil: primeiro passos rumo ao alcance das metas. Brasília: MS, 2018; 49(11):118.

6. BRASIL. MINISTÉRIO DA SAÚDE. Boletim Epidemiológico- Indicadores prioritários para o monitoramento do Plano Nacional pelo Fim da Tuberculose como Problema de Saúde Pública no Brasil. Brasília: MS, 2017b; 48(8):1-11.

7. BRASIL. MINISTÉRIO DA SAÚDE. Boletim Epidemiológico-Brasil Livre da Tuberculose: evolução dos cenários epidemiológicos e operacionais da doença. Brasília: MS, 2019a, 18 p.

8. BRASIL. MINISTÉRIO DA SAÚDE. Brasil Livre da Tuberculose: Plano Nacional pelo Fim da Tuberculose como Problema de Saúde Pública. Brasília: MS, 2017a. 54 p.

9. BRASIL. MINISTÉRIO DA SAÚDE. Rede de Teste Rápido para Tuberculose no Brasil: primeiro nano da implantação. Brasília: MS, 2015.

10. CAMPOS R, PIANTA C. Tuberculose: histórico, epidemiologia e imunologia, de 1990 a 1999, e co-infecção TB/HIV, de 1998 a 1999, Rio Grande do Sul-Brasil. Boletim da Saúde, 2001; 15(1):61-71.

11. CYRIACO C, et al. Programa Nacional de Controle da Tuberculose (PNCT). Controle da Tuberculose: uma proposta de integração ensino-serviço. Rio de Janeiro: Ed: ENSP/FIOCRUZ; 2014; 340 p.

12. JUNGES JR, et al. Tratamento Diretamente Observado da tuberculose: análise crítica da descentralização. Interface, 2019; 24:1-13.

13. KAPLAN RS, NORTON DP. Organização orientada para a estratégia: como as empresas que adotam o balancedscorecard prosperam no novo ambiente de negócios. Rio de Janeiro: Campus, 2000; 426p.

14. KRITSKI A, et al. Rede Brasileira de Pesquisa em Tuberculose-REDE TB. Anais do Instituto de Higiene e Medicina Tropical, 2018; 15:35-44.

15. MACIEL ELN, et al. O Brasil pode alcançar os novos objetivos globais da Organização Mundial da Saúde para o controle da tuberculose? Epidemiologia e Serviços de Saúde, 2018; 27(2):e0200007;1-4.

16. MACIEL ELN. Estratégias da agenda pós-2015 para o controle da tuberculose no Brasil: desafios e oportunidades. Epidemiologia e Serviços de Saúde, 2016; 25:423-426.

17. MACIEL MS, et al. A história da tuberculose no Brasil: os muitos tons (de cinza) da miséria. Revista da Sociedade Brasileira de Clínica Médica, 2012; 10(3):226-30.

18. MARQUES AMC, CUNHA RV. A medicação assistida e os índices de cura de tuberculose e de abandono de tratamento na população indígena Guaraní-Kaiwá no Município de Dourados, Mato Grosso do Sul, Brasil. Cadernos de Saúde Pública, 2003; 19(5):1405-11.

19. MISHIMA EO, NOGUEIRA PA. Tuberculose no idoso: estado de São Paulo, 1940-1995. Boletim de Pneumologia Sanitária, 2001; 9(1):5-11.

20. OLIVEIRA NF, GONÇALVES MJF. Fatores associados à hospitalização de pacientes com tuberculose. Revista LatinoAmericana de Enfermagem, 2013; 21(2):1-8. 
21. PEREIRA AA, et al. Gestão e gerenciamento dos níveis hierárquicos do programa nacional de controle da tuberculose. Interdisciplinary Journal of Health Education,2016; 1(1): 68-71.

22. PINTO ESG, et al. Articulação da saúde com a comunidade no manejo clínico da tuberculose. Revista Brasileira de Enfermagem, 2018; 71(3): 1122-1127.

23. ROSEMBERG J. Tuberculose - aspectos históricos, realidades, seu romantismo e transculturação. Boletim de PneumologiaSanitária, 1999;7(2):5-29.

24. RUFFINO-NETTO A, PEREIRA JC. Mortalidade por tuberculose e condições de vida: o caso Rio de Janeiro. Revista Saúde em Debate, 1981;12(1):27-34.

25. RUFFINO-NETTO A. Tuberculose: a calamidade negligenciada. Revista da Sociedade Brasileira de Medicina Tropical, 2002; 35(1):51-58.

26. SANTOS FILHO ET, GOMES ZMS. Estratégias de controle da tuberculose no Brasil: articulação e participação da sociedade civil. Revista de Saúde Pública, 2007;41(Supl. 1):111-116.

27. SEVERO NPF, et al. Características clínico-demográficas de pacientes hospitalizados com tuberculose no Brasil, no período de 1994 a 2004. Jornal Brasileiro de Pneumologia, 2007; 33 (5):565-571.

28. SHEPPARD DS. A literatura médica brasileira sobre a peste branca:1870-1940. História, Ciências, SaúdeBiomanguinhos, 2001; 8(1):172-92.

29. SILVA DR, et al. Série tuberculose 2019. Jornal Brasileiro de Pneumologia, 2019; 45(2): 1-2.

30. SOUZA EP, et al. Prevenção e controle da tuberculose: revisão integrativa da literatura. Revista Cuidarte, 2015; 6(2):1094-102.

31. SOUZA KMJ, et al. Abandono do tratamento de tuberculose e relações de vínculo com a equipe de saúde da família. Revista da Escola de Enfermagem da USP, 2010; 44(4):904-11.

32. WORLD HEALTH ORGANIZATION (WHO). Bending the curve - ending TB: Annual report 2017. Índia: World Health Organization, 2017.

33. WORLD HEALTH ORGANIZATION (WHO). Global tuberculosis report. [Internet]. 2016: World Health Organization; 2016.

34. WORLD HEALTH ORGANIZATION (WHO). Global Tuberculosis Report. [Internet]. 2014: World Health Organization; 2014.

35. WORLD HEALTH ORGANIZATION (WHO). Global tuberculosis report. [Internet]. 2019: World Health Organization, 2019. 